\title{
PICK'S FORMULA AND GENERALIZED EHRHART QUASI-POLYNOMIALS
}

\author{
Takayuki Hibi ${ }^{1}$, Miyuki Nakamura ${ }^{2}$, and Ivana Natalia \\ Kristantyo Samudro ${ }^{3}$, Akiyoshi Tsuchiya ${ }^{4}$ \\ ${ }^{1}$ Department of Pure and Applied Mathematics, Graduate School of \\ Information Science and Technology, Osaka University, \\ Toyonaka, Osaka 560-0043, Japan \\ hibi@math.sci.osaka-u.ac.jp \\ ${ }^{2}$ Department of Pure and Applied Mathematics, Graduate School of \\ Information Science and Technology, Osaka University, \\ Toyonaka, Osaka 560-0043, Japan \\ u510908d@ecs.osaka-u.ac.jp \\ ${ }^{3}$ Department of Pure and Applied Mathematics, Graduate School of \\ Information Science and Technology, Osaka University, \\ Toyonaka, Osaka 560-0043, Japan \\ u564297d@ecs.osaka-u.ac.jp \\ ${ }^{4}$ Department of Pure and Applied Mathematics, Graduate School of \\ Information Science and Technology, Osaka University, \\ Toyonaka, Osaka 560-0043, Japan \\ a-tsuchiya@cr.math.sci.osaka-u.ac.jp
}

\begin{abstract}
By virtue of Pick's formula, the generalized Ehrhart quasi-polynomial of the triangulation $\mathcal{P}(n) \subset \mathbb{R}^{2}$ with the vertices $(0,0),(u(n), 0),(0, v(n))$, where $u(x)$ and $v(x)$ belong to $\mathbb{Z}[x]$ and where $n=1,2, \ldots$, will be computed.

Key words: Generalized Ehrhart quasi-polynomial, Pick's formula.
\end{abstract}

\begin{abstract}
Abstrak. Dengan menggunakan formulasi Pick, disajikan perhitungan dari polinomial quasi Ehrhart yang diperumum dari triangulasi $\mathcal{P}(n) \subset \mathbb{R}^{2}$ dengan verteks $(0,0),(u(n), 0),(0, v(n))$ dimana $u(x), v(x) \in \mathbb{Z}[x]$ dan $n=1,2, \ldots$

Kata kunci: Generalized Ehrhart quasi-polynomial, Pick's formula.
\end{abstract}

2010 Mathematics Subject Classification: 52B20 


\section{Introduction}

The enumeration of the integer points belonging to a rational convex polytope is one of the most traditional topics in combinatorics.

Let $\mathbb{Z}_{\geq 0}$ denote the set of nonnegative integers. Recall that a numerical function $f: \mathbb{Z}_{\geq 0} \rightarrow \mathbb{Z}_{\geq 0}$ is a quasi-polynomial if there exist an integer $s \geq 1$, called a period of $f$, and polynomials $f_{0}(x), \ldots, f_{s-1}(x)$ belonging to $\mathbb{Q}[x]$ such that $f(n)=f_{i}(n)$ when $n \equiv i(\bmod s)$. Furthermore, the quasi-period of $f$ is the smallest integer $r \geq 1$ such that there exist subsets $A_{1}, \ldots, A_{r}$ of $\mathbb{Z}_{\geq 0}$ with $\mathbb{Z}_{\geq 0}=A_{1} \cup \cdots \cup A_{r}$ and polynomials $g_{1}(x), \ldots, g_{r}(x)$ belonging to $\mathbb{Q}[x]$ for which $f(n)=g_{i}(n)$ when $n \in A_{i}$.

A typical example of a quasi-polynomial is the function $\sharp\left(n \mathcal{P} \cap \mathbb{Z}^{d}\right)$, called the Ehrhart quasi-polynomial ([1], [3]), arising from a rational convex polytope $\mathcal{P} \subset \mathbb{R}^{d}$.

More generally, given polynomials $w_{i}^{(j)}(x) \in \mathbb{Z}[x], 1 \leq i \leq q$ and $1 \leq j \leq d$, we introduce $v_{i}(n) \in \mathbb{Z}^{d}, n=1,2, \ldots$, by setting $v_{i}(n)=\left(w_{i}^{(1)}(n), \ldots, w_{i}^{(d)}(n)\right)$. Write $\mathcal{P}_{\left\{w_{i}^{(j)}\right\}}(n) \subset \mathbb{R}^{d}$ for the convex polytope which is the convex hull of $\left\{v_{1}(n), \ldots, v_{q}(n)\right\}$. It follows from [2] that the numerical function $\sharp\left(\mathcal{P}_{\left\{w_{i}^{(j)}\right\}}(n) \cap \mathbb{Z}^{d}\right)$ is a quasi-polynomial, which is called the generalized Ehrhart quasi-polynomial of $\left\{\mathcal{P}_{\left\{w_{i}^{(j)}\right\}}(n)\right\}_{n=1,2, \ldots}$.

We now come to a basic problem which we are interested in. Let $\mathbf{0}$ be the origin of $\mathbb{R}^{d}$ and $\mathbf{e}_{1}, \ldots, \mathbf{e}_{d}$ the canonical unit coordinate vectors of $\mathbb{R}^{d}$.

Problem 1. Given arbitrary integers $r \geq 1, e \geq 1$ and $d \geq 2$, find polynomials $v_{1}(x), \ldots, v_{d}(x)$ belonging to $\mathbb{Z}[x]$ with each $\operatorname{deg}\left(v_{i}(x)\right)=e$ such that the quasiperiod of the generalized Ehrhart quasi-polynomial $\sharp\left(\mathcal{P}(n) \cap \mathbb{Z}^{d}\right)$ of $\{\mathcal{P}(n)\}_{n=1,2, \ldots}$ is $r$, where $\mathcal{P}(n) \subset \mathbb{R}^{d}$ is the simplex with the vertices $\mathbf{0}, v_{1}(n) \mathbf{e}_{1}, \ldots, v_{d}(n) \mathbf{e}_{d}$.

In the present paper, by virtue of Pick's formula, an answer to Problem 1 for $d=2$ can be given.

\section{MAIN RESULT}

The following theorem is the main result in this paper.

Theorem 2. Given arbitrary integers $r \geq 1, e \geq 1$ and $s \geq 1$, there exist polynomials $u(x)$ and $v(x)$ belonging to $\mathbb{Z}[x]$ with $\operatorname{deg}(u(x))=\operatorname{deg}(v(x))=e$ for which the quasi-period of the generalized Ehrhart quasi-polynomial $\sharp\left(\mathcal{P}(n) \cap \mathbb{Z}^{2}\right)$ is r, where $\mathcal{P}(n) \subset \mathbb{R}^{2}$ is a triangle with the vertices $(0,0),(u(n), 0),(0, v(n))$, and the smallest period of $\sharp\left(\mathcal{P}(n) \cap \mathbb{Z}^{2}\right)$ is bigger than $s$.

Proof. Fix a prime number $p>1$. Let $u(x)=x^{e}$ and $v(x)=x^{e}+p^{e(r-1)}$. Write $A(\mathcal{P}(n))$ for the area of $\mathcal{P}(n)$. Let $I(\mathcal{P}(n))$ and $B(\mathcal{P}(n))$ denote the number of integer points belonging to the interior of $\mathcal{P}(n)$ and the number of integer points belonging to the boundary of $\mathcal{P}(n)$, respectively. Pick's formula guarantees that

$$
A(\mathcal{P}(n))=I(\mathcal{P}(n))+\frac{1}{2} B(\mathcal{P}(n))-1 .
$$


Moreover, one has $A(\mathcal{P}(n))=u(n) v(n) / 2$. Let $f(n)=\sharp\left(\mathcal{P}(n) \cap \mathbb{Z}^{2}\right)$. Since $f(n)=$ $I(\mathcal{P}(n))+B(\mathcal{P}(n))$, it follows that

$$
f(n)=\frac{1}{2} u(n) v(n)+\frac{1}{2} B(\mathcal{P}(n))+1 .
$$

Let $\mathcal{H}(n) \subset \mathbb{R}^{2}$ denote the segment which is the convex hull of $\{(u(n), 0),(0, v(n))\}$ and $g(n)=\sharp\left(\mathcal{H}(n) \cap \mathbb{Z}^{2}\right)$. Since $B(\mathcal{P}(n))=g(n)+u(n)+v(n)-1$, it follows that

$$
f(n)=\frac{1}{2}(u(n) v(n)+u(n)+v(n)+1)+\frac{1}{2} g(n) .
$$

Now, what we must show is that the quasi-period of the quasi-polynomial $g(n)$ is equal to $r$. One has

$$
g(n)=\sharp\left\{(x, y) \in \mathbb{Z}_{\geq 0}^{2}: \frac{x}{u(n)}+\frac{y}{v(n)}=1\right\} .
$$

Let $h(n)$ denote the greatest common divisor of $u(n)=n^{e}$ and $v(n)=n^{e}+p^{e(r-1)}$. In other words, $h(n)$ is the greatest common divisor of $n^{e}$ and $p^{e(r-1)}$. Writing $u(n)=h(n) u_{0}(n)$ and $v(n)=h(n) v_{0}(n)$, it follows that

$$
g(n)=\sharp\left\{(x, y) \in \mathbb{Z}_{\geq 0}^{2}: \frac{x}{u_{0}(n)}+\frac{y}{v_{0}(n)}=h(n)\right\} .
$$

Since $u_{0}(n)$ and $v_{0}(n)$ are relatively prime, one has $g(n)=h(n)+1$. We claim that the quasi-period of the quasi-polynomial $h(n)$ is equal to $r$. Let $k$ denote the biggest integer for which $n$ is divided by $p^{k}$. Then

- if $k=0$, then $n^{e}$ and $p^{e(r-1)}$ are relatively prime and $h(n)=1$;

- if $1 \leq k \leq r-2$, then $h(n)=p^{e k}$;

- if $k \geq r-1$, then $h(n)=p^{e(r-1)}$.

Thus the quasi-period of $h(n)$ is equal to $r$, as desired.

We claim that the smallest period of $h(n)$ is $p^{r-1}$. Let $n \equiv b\left(\bmod p^{r-1}\right)$, where $0 \leq b<p^{r-1}$. When $b=0$, one has $h(n)=p^{e(r-1)}$. Let $1 \leq b<p^{r-1}$ and $\ell$ the biggest integer for which $n$ is divided by $p^{\ell}$, where $0 \leq \ell \leq r-2$. When $\ell=0$, one has $h(n)=1$. When $1 \leq \ell \leq r-2$, one has $h(n)=p^{e k}$. Hence the smallest period of $h(n)$ is $p^{r-1}$. Finally, if $p$ is large enough, then the smallest period of $h(n)$ is bigger than $s$, as required.

\section{EXAMPLES}

As the end of this paper, we give some examples.

In Theorem 2, when $s \geq r$, it would, of course, be of interest to find $u(x)$ and $v(x)$ belonging to $\mathbb{Z}[x]$ for which the quasi-period of the generalized Ehrhart quasi-polynomial $\sharp\left(\mathcal{P}(n) \cap \mathbb{Z}^{2}\right)$ is $r$ and the smallest period of $\sharp\left(\mathcal{P}(n) \cap \mathbb{Z}^{2}\right)$ is $s$. 
Example 3. Let $e=3, r=4$ and $p=2$ in the proof of Theorem 2. Thus $u(x)=x^{3}$ and $v(x)=x^{3}+2^{9}$. Then

$$
\sharp\left(\mathcal{P}(n) \cap \mathbb{Z}^{2}\right)= \begin{cases}\frac{n^{6}}{2}+2^{8}\left(n^{3}+1\right)+n^{3}+\frac{3}{2}, & n: \text { odd, } \\ \frac{n^{6}}{2}+2^{8}\left(n^{3}+1\right)+n^{3}+\frac{1}{2}+2^{2}, & n=2 \cdot a, \\ \frac{n^{6}}{2}+2^{8}\left(n^{3}+1\right)+n^{3}+\frac{1}{2}+2^{5}, & n=2^{2} \cdot a, \\ \frac{n^{6}}{2}+2^{8}\left(n^{3}+1\right)+n^{3}+\frac{1}{2}+2^{8}, & n=2^{k} \cdot a, k \geq 3,\end{cases}
$$

where $a \geq 1$ is an odd integer. Furthermore,

$$
\sharp\left(\mathcal{P}(n) \cap \mathbb{Z}^{2}\right)= \begin{cases}\frac{n^{6}}{2}+2^{8}\left(n^{3}+1\right)+n^{3}+\frac{3}{2}, & n \equiv 1,3,5,7(\bmod 8), \\ \frac{n^{6}}{2}+2^{8}\left(n^{3}+1\right)+n^{3}+\frac{1}{2}+2^{2}, & n \equiv 2,6(\bmod 8), \\ \frac{n^{6}}{2}+2^{8}\left(n^{3}+1\right)+n^{3}+\frac{1}{2}+2^{5}, & n \equiv 4(\bmod 8), \\ \frac{n^{6}}{2}+2^{8}\left(n^{3}+1\right)+n^{3}+\frac{1}{2}+2^{8}, & n \equiv 0(\bmod 8) .\end{cases}
$$

Thus the quasi-period of the quasi-polynomial $\sharp\left(\mathcal{P}(n) \cap \mathbb{Z}^{2}\right)$ is equal to 4 , while its smallest period is 8 .

Example 4. Let $u(x)=x^{2}+3 x+2$ and $v(x)=x^{2}+4 x+1$. Write $h(n)$ for the greatest common divisor of $u(n)$ and $v(n)$. Let $u(n)=h(n) u_{0}(n)$ and $v(n)=$ $h(n) v_{0}(n)$. Then $n=h(n)\left(v_{0}(n)-u_{0}(n)\right)+1$. Thus

$$
h(n)\left(u_{0}(n)-h(n)\left(v_{0}(n)-u_{0}(n)\right)^{2}-5\left(v_{0}(n)-u_{0}(n)\right)\right)=6 .
$$

Hence $h(n) \in\{1,2,3,6\}$. A routine computation shows that

$$
h(n)= \begin{cases}1, & n=6 k-4 \text { or } 6 k, \\ 2, & n=6 k-3 \text { or } 6 k-1, \\ 3, & n=6 k-2, \\ 6, & n=6 k-5 .\end{cases}
$$

Following the proof of Theorem 2, one has

$$
\sharp\left(\mathcal{P}(n) \cap \mathbb{Z}^{2}\right)= \begin{cases}\frac{n^{4}}{2}+\frac{7 n^{3}}{2}+\frac{17 n^{2}}{2}+9 n+4, & n=6 k-4 \text { or } 6 k, \\ \frac{n^{4}}{2}+\frac{7 n^{3}}{2}+\frac{17 n^{2}}{2}+9 n+\frac{9}{2}, & n=6 k-3 \text { or } 6 k-1, \\ \frac{n^{4}}{2}+\frac{7 n^{3}}{2}+\frac{17 n^{2}}{2}+9 n+5, & n=6 k-2, \\ \frac{n^{4}}{2}+\frac{7 n^{3}}{2}+\frac{17 n^{2}}{2}+9 n+\frac{13}{2}, & n=6 k-5 .\end{cases}
$$


Thus the quasi-period of the quasi-polynomial $\sharp\left(\mathcal{P}(n) \cap \mathbb{Z}^{2}\right)$ is equal to 4 , while its smallest period is 6 .

\section{REFERENCES}

[1] Beck, M., and Robins, S., Computing the continuous discretely, Springer, 2007.

[2] Chen, S., Li, N., and Sam, S.V., "Generalized Ehrhart polynomials", Trans. Amer. Math. Soc. 364(2012), 551-569.

[3] Stanley, R.P., Enumerative Combinatorics, Volume I, Second Ed., Cambridge University Press, 2012. 
T. HiBI, et al. 